\title{
The HER2 Receptor in Breast Cancer: Pathophysiology, Clinical Use, and New Advances in Therapy
}

\author{
Zahi Mitri, ${ }^{1}$ Tina Constantine, ${ }^{1}$ and Ruth O'Regan ${ }^{2,3}$ \\ ${ }^{1}$ Department of Internal Medicine, Emory University, Atlanta, GA 30322, USA \\ ${ }^{2}$ Department of Hematology \& Medical Oncology, Emory University, Atlanta, GA 30322, USA
}

${ }^{3}$ Winship Cancer Institute, Emory University, Atlanta, GA 30322, USA

Correspondence should be addressed to Ruth O’Regan, rmoregan23@gmail.com

Received 10 June 2012; Accepted 26 November 2012

Academic Editor: Nabil F. Saba

Copyright (c) 2012 Zahi Mitri et al. This is an open access article distributed under the Creative Commons Attribution License, which permits unrestricted use, distribution, and reproduction in any medium, provided the original work is properly cited.

\begin{abstract}
Human epidermal growth factor receptor 2 (HER2) is overexpressed in around 20-30\% of breast cancer tumors. It is associated with a more aggressive disease, higher recurrence rate, and increased mortality. Trastuzumab is a HER2 receptor blocker that has become the standard of care for the treatment of HER2 positive breast cancer. The effectiveness of Trastuzumab has been well validated in research as well as in clinical practice. The addition of Trastuzumab to standard of care chemotherapy in clinical trials has been shown to improve outcomes for early stage as well as metastatic HER2 positive breast cancer. The most clinically significant side effect of Trastuzumab is the risk of cardiac myocyte injury, leading to the development of congestive heart failure. The emergence of patterns of resistance to Trastuzumab has led to the discovery of new monoclonal antibodies and other targeted agents aimed at overcoming Trastuzumab resistance and improving survival in patients diagnosed with HER2 positive breast cancers.
\end{abstract}

\section{Introduction}

Human epidermal growth factor receptor HER2 overexpression is present in approximately $20-30 \%$ of breast cancer tumors. HER2 overexpression is associated with a more aggressive disease, higher recurrence rate, and shortened survival [1-4]. Trastuzumab is a humanized monoclonal antibody targeting the HER2 receptor, which was approved for use in 1998. The mechanisms of action of Trastuzumab have not been clearly defined, but likely include extracellular mechanisms involving antibody-dependent cellular cytotoxicity (ADCC), and intracellular mechanisms involving apoptosis and cell cycle arrest as well as inhibiting angiogenesis, and preventing DNA repair following chemotherapyinduced damage $[5,6]$. Trastuzumab has been shown to be effective in combination with chemotherapy, for the treatment of early stage and metastatic HER2 positive breast cancer.

\section{Biology}

HER2 is part of the epidermal growth factor (EGF) family, along with 3 other receptors: epidermal growth factor receptor (HER1, erbB1), HER2 (erbB2), HER3 (erbB3), and HER4 (erbB4). The HER2 gene is located on the long arm of chromosome 17 and encodes a $185-\mathrm{kDa}$ transmembrane protein [7-9]. The HER2 receptor extracellular domain has no identifiable ligand, unlike the other EGF family receptors. It is present in an active conformation and can undergo ligand-independent dimerization with other EGF receptors $[6,10,11]$. The most active and tumor promoting combination is thought to be the HER2/HER3 dimer [12-15].

The mechanism of action of Trastuzumab is perceived to be through both innate and adaptive immunities. Innate mechanisms lead to cell cycle arrest, with a noted increase in p27 levels, and decrease in cyclin D1 and cyclin-dependent kinase 2 activity [16]. Trastuzumab alone does not seem to promote a significant level of apoptosis, but is synergistic with most chemotherapeutics in preclinical models. This synergism is felt in part to be explained by inhibition of the PI3K/Akt signaling pathway, which normally promotes cell survival [17]. Nevertheless, the innate response alone does not fully explain the effect of Herceptin on tumor regression. Adaptive mechanisms are also present and involve antibodydependent cell-mediated cytotoxicity (ADCC). This is likely 
to be T-cell mediated, through activation of the FC receptor, leading to increased cell death [18].

\section{Clinical Use}

Trastuzumab has become the standard of care in the treatment of patient with HER2 positive breast cancer. Data from several randomized trials demonstrated that the addition of Trastuzumab to chemotherapy regimens in the adjuvant setting improves outcome in women with early stage breast cancer [19-22]. In the NSABP B31-NCCTG joint analysis, adjuvant therapy with Trastuzumab for primary operable HER2 positive breast cancer decreases recurrence rates and improves overall survival with a 5-year follow-up analysis [23]. The BCIRG 006 trial showed that the addition of 52 weeks of Trastuzumab therapy to chemotherapy was effective in prolonging disease-free survival and overall survival in patients with early stage HER 2 positive breast cancer. This effect was seen with both anthracycline and nonanthracycline-based chemotherapy regimens [24]. In the HERA trial, one year of Trastuzumab following standard chemotherapy was effective in prolonging disease-free and overall survival, at a four-year followup [25]. It is worth noting that patients who crossed over from the observation group to the Trastuzumab arm were also noted to have improved survival. Lastly, the FinHer trial demonstrated that Trastuzumab added to 9-weeks of chemotherapy significantly improved recurrence survival when compared to chemotherapy alone [26].

The ideal timing of Trastuzumab administration appears to be concurrent administration with chemotherapy, based on the NSABP trial [22]. However, this remains controversial since the HERA trial continues to show a benefit for sequential Trastuzumab with a followup of 4 years [25]. In the United States, concurrent chemotherapy and Trastuzumab are most commonly used [27].

Trastuzumab has also been shown to be effective in improving outcome for patients with HER2 positive metastatic breast cancer [28]. This effect has been validated in multiple trials [17, 29]. The Hercules trial [30] showed that combining Trastuzumab with epirubicin and cyclophosphamide resulted in improved response to therapy and prolonged the time to disease progression. The addition of Trastuzumab was also effective in improving survival when used with different chemotherapy regimens, such as docetaxel in the M77001 trial [31]. In the HERTAX trial, the combination of Trastuzumab and docetaxel allowed increased time to disease progression in a higher proportion of patients when compared with single-agent Trastuzumab followed by single-agent docetaxel at disease progression [32]. The HERNATA study again showed that a combination of Trastuzumab with paclitaxel or docetaxel as first line therapy for HER2 positive metastatic breast cancer improved patient overall survival and median time to disease progression [33]. In the BCIRG 007, both regimens (Docetaxel and Trastuzumab v/s Docetaxel, Carboplatin and Trastuzumab) were highly effective in prolonging time to progression of disease, overall survival, and both had acceptable safety profiles [34]. There has also been data that Trastuzumab may be a safe and effective single agent as first-line treatment for HER2 positive metastatic breast cancer [35].

Trastuzumab has been evaluated in combination with hormonal therapy in postmenopausal women with metastatic breast cancer, that is, both hormone receptor and HER2positive. The TAnDEM study showed that the combination of anastrozole and Trastuzumab was more effective in improving outcomes when compared with anastrozole alone [36].

Trastuzumab has been evaluated extensively in the neoadjuvant setting. A review by Lazaridis et al. presented positive data regarding the integration of Trastuzumab into neoadjuvant treatment regimens [37]. The follow-up results from the GeparQuattro study revealed that higher pathological clinical response was achieved when Trastuzumab was added to neoadjuvant chemotherapy, whether it is an anthracyclines- or taxane-based regimen [38]. These results were validated in other studies, as addition of neoadjuvant Trastuzumab therapy appears to be effective in achieving a higher rate of remission [39-41]. In the NOAH trial, the addition of Trastuzumab to neoadjuvant chemotherapy, followed by 52 weeks of Trastuzumab, was effective in improving event-free survival in patients with HER2-positive locally advanced or inflammatory breast cancer [42].

Lastly, a trial by Buzdar et al. evaluated the effect of adding Trastuzumab to chemotherapy in the neoadjuvant setting for operable HER2 positive breast cancer. The trial was stopped early due to significantly higher rates of pathological response in the Trastuzumab arm [39]. Pathologic complete response appears to be a surrogate marker of outcome for HER2-positive breast cancers, especially those that are ER-negative. There still remain questions to be answered as to the duration, optimal chemotherapy regimen to be used with Trastuzumab therapy, and whether chemotherapy is necessary in combination with Trastuzumab for all patients in the neoadjuvant setting.

\section{Cardiotoxicity}

Cardiac toxicity in patients receiving anthracycline-based chemotherapy has been well established. The mechanism of doxorubicin cardiotoxicity involves direct injury to cardiac myocytes through increased free radicals and oxidative stress [43-45]. Doxorubicin cardiotoxicity is mostly irreversible, which clinically leads to decreased left ventricular ejection fraction (LVEF) and subsequently congestive heart failure symptoms. Due to causing structural damage to the myocytes, doxorubicin therapy renders the myocardium more susceptible to injury and irreversible defects from subsequent stressors, whether their insult is reversible or not $[46,47]$.

Trastuzumab therapy, unlike anthracyclines, has been associated with reversible injury to the cardiac myocytes. This is mainly due to the fact that it does not appear to cause structural damage to the myocardium [48]. Human epidermal growth factor receptor-2 (HER2/ERB2) is expressed in the adult myocardium and is believed to modulate cardiac function and anthracycline cardiotoxicity [49]. Neuregulin-1 (NRG-1) is a protein essential to cell cycle survival. It triggers 
a cascade of events that promotes sarcomere stability and relieves oxidative stress $[50,51]$.

HER2 binds to Neuregulin-1 and initiates this cascade, perpetuating signals to maintain cell survival, response to stress, and prevent apoptosis. Trastuzumab blocks the HER2 receptor, causing these pathways to be interrupted [52-56]. The mechanism of Trastuzumab-induced cardiac toxicity has not clearly been elucidated yet. It is proposed to be a combination of increased oxidative stress as well as inhibiting antiapoptotic mechanisms [57]. The increased oxidative stress seems to occur due to the inhibition of NADPH oxidase, as well as upregulation of the angiotensin II pathways, which in turn cause further inhibition of NRG-1 pathways. All of these events cause an increase in free radicals and subsequently myocyte injury $[58,59]$. In addition to that, studies have shown that blockade of the HER2 receptor with Trastuzumab leads to an increased ratio of proapoptotic to antiapoptotic proteins. This leads to shortened cell survival and accelerates apoptosis [60]. These findings, however, do not explain the difference in cardiotoxicity between Trastuzumab and Doxorubicin. Further studies have shown that inhibition of the HER2 receptor leads to changes in the tertiary structure of the cardiac contractile apparatus, but does not induce myocardial cell death. This would explain the transient decline in cardiac function during treatment with Trastuzumab, and recovery with interruption of therapy. This is in contrast to anthracycline toxicity which induces nonreversible changes through maladaptive cardiac remodeling, progressing eventually to heart failure [61-63]. Combined therapy with anthracyclines and Trastuzumab affects a common pathway crucial to cell survival and dealing with oxidative stress. Despite the fact that Trastuzumabinduced myocardial injury is largely reversible, it leaves myocytes more vulnerable to anthracycline-induced toxicity. With the survival pathways inhibited and potentiated by two drugs, cell survival is put at risk, and there is increased risk of apoptosis. This reflects clinically in myocyte death and depressed LVEF, leading to signs and symptoms of CHF [57]. The criteria for Trastuzumab-induced cardiotoxicity was defined in 2002 by The Cardiac Review and Evaluation Committee (CREC). These included (1) cardiomyopathy characterized by a decrease in the left ventricular ejection fraction (LVEF) that is global or more severe in the septum; (2) symptoms of CHF; (3) associated signs of CHF including but not limited to S3 gallop, tachycardia, or both; and (4) a decline in LVEF of $5 \%$ to $<55 \%$ with accompanying signs or symptoms of CHF, or a decline in LVEF of $\geq 10 \%$ to $<55 \%$ without accompanying signs or symptoms. The presence of any one symptom is enough for the diagnosis of cardiac dysfunction [64]. Trastuzumab has been shown to be associated with improved outcomes in treatment of HER2 positive breast cancer, whether early or metastatic [21]. Several studies have, however, confirmed that there is a higher incidence of cardiac toxicity associated with the use of Trastuzumab. The highest risk seems to occur in the setting of anthracycline therapy followed by Trastuzumab. The combination of a taxane-based regimen followed by Trastuzumab appears to cause less cardiac dysfunction $[17,28,31,65]$. The myocardial injury associated with Trastuzumab was found to be reversible in patients who were followed for a long period of time after interruption of treatment. Patients were able to recover their LVEF to their pretreatment baseline, and clinical evidence of CHF was resolved $[27,49,63]$.

Certain risk factors have been associated with increased risk of cardiac dysfunction with Trastuzumab treatment. These included age $>50$ years, concomitant use of an anthracycline, hypertension requiring treatment, and post-AC LVEF values of $50-54 \%$ [64]. In addition to that, elevation in Troponin levels during the course of anthracycline and Trastuzumab therapy was found to be a strong predictor of subsequent decrease in cardiac function and LVEF. With respect to Trastuzumab therapy, the Troponin rise was seen early on, and affected patients were less likely to regain baseline cardiac function [66, 67]. Given the proven benefit of Trastuzumab therapy with HER2 over expression, research focused on looking for interventions that would help to prevent treatment-induced cardiac toxicity. Researchers first examined nonpharmacological interventions such as aerobic exercise. This was not found to have any benefit in preventing remodeling and cardiac dysfunction associated with Trastuzumab [68-70]. Studies have shown that beta blocker therapy (carvedilol) and angiotensin converting enzyme inhibitors were effective in preventing decrease in LVEF in patients on anthracycline therapy [67, 71]. MANTICORE 101 is an ongoing trial which aims to examine the effect of approved CHF therapy (betablocker, ACEi) on preventing cardiac dysfunction in the setting of Trastuzumab therapy [72].

\section{Trastuzumab Resistance}

Trastuzumab has become widely used for the treatment of HER2 positive breast cancer. Despite the success of Trastuzumab-based therapy in treating all stages of HER2positive breast cancer, resistance to Trastuzumab is an important issue which affects outcome for a subset of patients. These observations have prompted research into the mechanisms of Trastuzumab resistance, which are thought to underlie failure of therapy [35]. No clear etiology has been identified, but several hypotheses have emerged. First of all, studies have found a truncated HER2 receptor, lacking an extracellular domain, which prevents Trastuzumab binding, hence making it ineffective. Interestingly, the same cell line was successfully inhibited by a combined EGFR/HER2 inhibitor, lapatinib $[5,73,74]$.

Lapatinib is a dual EGFR and HER2 receptor tyrosine kinase inhibitor $[75,76]$. Lapatinib has been shown to be effective in research in inhibiting growth of breast cancer lines known to be resistant to Trastuzumab [77]. In patients who had documented disease progression on Trastuzumab, the addition of Lapatinib in combination with chemotherapy [78] or with Trastuzumab [79] was effective in prolonging time to disease progression and overall survival. The addition of lapatinib to letrozole for the treatment of hormone positive HER2 positive metastatic breast cancer was superior to letrozole alone in prolonging progressionfree survival [80]. Lapatinib is approved in combination with capecitabine for patients with HER2-positive metastatic 
breast cancer previously treated with Trastuzumab, and in combination with letrozole for metastatic breast cancer that is both hormone receptor and HER2-positive. Pertuzumab is another monoclonal antibody that targets HER2 but attaches to a different site on the receptor and, as such, inhibits heterodimerization between HER2 and other Her receptors [81]. The Cleopatra study randomized patients with metastatic HER2 positive breast cancer to placebo plus Trastuzumab plus docetaxel (control group) or pertuzumab plus Trastuzumab plus docetaxel. The median progression free survival was longer in the pertuzumab group [82, 83]. Pertuzumab is now approved in combination with docetaxel and Trastuzumab for the first-line treatment of HER2-positive metastatic breast cancer. Trastuzumab emtansine (Trastuzumab-DM1) is a new product in which the Trastuzumab moiety binds to HER2 on tumor cell surfaces and, upon internalization, the DM1 moiety is released and binds to tubulin, thereby disrupting microtubule assembly/disassembly dynamics and inhibiting cell division and proliferation. Primary results from the EMILIA study, a randomized trial comparing Trastuzumab DM-1 to lapatinib and capecitabine, have shown that Trastuzumab DM-1 significantly improved progression-free survival in patients with metastatic HER2-positive breast cancer. Further research is looking into inhibiting other targets such as mTOR, P13K, or AKT pathways. Two randomized trials are evaluating the addition of the mTOR inhibitor, everolimus, to chemotherapy and Trastuzumab in the first-line and Trastuzumab-resistance metastatic setting.

\section{Conclusion}

Trastuzumab has been proven to be effective in improving outcomes for patients with early operable as well as metastatic HER2 positive metastatic breast cancer. It has also been shown to increase the pathological response when used in the neoadjuvant setting. Trastuzumab therapy has been associated with an increased risk of cardiac toxicity, especially when used in combination with anthracyclines. Resistance to Trastuzumab therapy has also been documented, as patients have had disease progression while on treatment. A better understanding of the mechanisms of resistance to Trastuzumab therapy constitutes the cornerstone of finding new better tailored therapeutic agents capable of reducing rates of treatment failure and disease progression. Multiple new drugs are under investigation, targeting a wide array of receptors and cell cycle regulatory proteins. Preclinical pharmacological and genomic data will soon make its way into the clinical domain. Integrating this knowledge into therapeutic decision making may change the identity of HER2 positive breast cancer from a one drug for all diseases into a personalized individualized therapy, tailored to each patient's predictable response.

\section{References}

[1] C. A. Hudis, "Trastuzumab-mechanism of action and use in clinical practice," The New England Journal of Medicine, vol. 357, no. 1, pp. 39-51, 2007.
[2] F. Meric-Bernstam and M. C. Hung, "Advances in targeting human epidermal growth factor receptor-2 signaling for cancer therapy," Clinical Cancer Research, vol. 12, no. 21, pp. 6326-6330, 2006.

[3] D. J. Slamon, G. M. Clark, and S. G. Wong, "Human breast cancer: correlation of relapse and survival with amplification of the HER-2/neu oncogene," Science, vol. 235, no. 4785, pp. $177-182,1987$.

[4] D. J. Slamon, W. Godolphin, L. A. Jones et al., "Studies of the HER-2/neu proto-oncogene in human breast and ovarian cancer," Science, vol. 244, no. 4905, pp. 707-712, 1989.

[5] M. Nuti, F. Bellati, V. Visconti et al., "Immune effects of trastuzumab," Journal of Cancer, vol. 2, pp. 317-323, 2011.

[6] N. L. Spector and K. L. Blackwell, "Understanding the mechanisms behind trastuzumab therapy for human epidermal growth factor receptor 2-positive breast cancer," Journal of Clinical Oncology, vol. 27, no. 34, pp. 5838-5847, 2009.

[7] S. I. Fukushige, K. I. Matsubara, and M. Yoshida, "Localization of a novel v-erbB-related gene, c-erbB-2, on human chromosome 17 and its amplification in a gastric cancer cell line," Molecular and Cellular Biology, vol. 6, no. 3, pp. 955-958, 1986.

[8] D. F. Stern, P. A. Heffernan, and R. A. Weinberg, "P185, a product of the neu proto-oncogene, is a receptorlike protein associated with tyrosine kinase activity," Molecular and Cellular Biology, vol. 6, no. 5, pp. 1729-1740, 1986.

[9] T. Akiyama, C. Sudo, and H. Ogawara, "The product of the human c-erbB-2 gene: a 185-kilodalton glycoprotein with tyrosine kinase activity," Science, vol. 232, no. 4758, pp. 16441646, 1986.

[10] A. K. Koutras and T. R. Evans, "The epidermal growth factor receptor family in breast cancer," Journal of OncoTargets and Therapy, vol. 1, pp. 5-19, 2008.

[11] D. Graus-Porta, R. R. Beerli, J. M. Daly, and N. E. Hynes, "ErbB-2, the preferred heterodimerization partner of all ErbB receptors, is a mediator of lateral signaling," The EMBO Journal, vol. 16, no. 7, pp. 1647-1655, 1997.

[12] M. Alimandi, A. Romano, M. C. Curia et al., "Cooperative signaling of ErbB3 and ErbB2 in neoplastic transformation and human mammary carcinomas," Oncogene, vol. 10, no. 9, pp. 1813-1821, 1995.

[13] R. Pinkas-Kramarski, A. E. G. Lenferink, S. S. Bacus et al., "The oncogenic ErbB-2/ErbB-3 heterodimer is a surrogate receptor of the epidermal growth factor and betacellulin," Oncogene, vol. 16, no. 10, pp. 1249-1258, 1998.

[14] C. Wallasch, F. U. Weiss, G. Niederfellner, B. Jallal, W. Issing, and A. Ullrich, "Heregulin-dependent regulation of HER2/ neu oncogenic signaling by heterodimerization with HER3," The EMBO Journal, vol. 14, no. 17, pp. 4267-4275, 1995.

[15] K. Zhang, J. Sun, N. Liu et al., "Transformation of $\mathrm{NIH}_{3} \mathrm{~T}_{3}$ cells by $\mathrm{HER}_{3}$ or $\mathrm{HER}_{4}$ receptors requires the presence of HER 1 or HER2," The Journal of Biological Chemistry, vol. 271, no. 7, pp. 3884-3890, 1996.

[16] T. Mukohara, "Mechanisms of resistance to anti-human epidermal growth factor receptor 2 agents in breast cancer," Cancer Science, vol. 102, no. 1, pp. 1-8, 2011.

[17] M. D. Pegram, G. E. Konecny, C. O’Callaghan, M. Beryt, R. Pietras, and D. J. Slamon, "Rational combinations of Trastuzumab with chemotherapeutic drugs used in the treatment of breast cancer," Journal of the National Cancer Institute, vol. 96, no. 10, pp. 739-749, 2004.

[18] S. Park, Z. Jiang, E. D. Mortenson et al., "The therapeutic effect of anti-HER2/neu antibody depends on both innate and adaptive immunity," Cancer Cell, vol. 18, no. 2, pp. 160-170, 2010. 
[19] Y. Madarnas, M. Trudeau, J. A. Franek, D. McCready, K. I. Pritchard, and H. Messersmith, "Adjuvant/neoadjuvant trastuzumab therapy in women with HER-2/neu-overexpressing breast cancer: a systematic review," Cancer Treatment Reviews, vol. 34, no. 6, pp. 539-557, 2008.

[20] G. Mariani, A. Fasolo, E. De Benedictis, and L. Gianni, "Trastuzumab as adjuvant systemic therapy for HER2-positive breast cancer," Nature Clinical Practice Oncology, vol. 6, no. 2, pp. 93-104, 2009.

[21] M. J. Piccart-Gebhart, M. Procter, B. Leyland-Jones et al., "Trastuzumab after adjuvant chemotherapy in HER2-positive breast cancer," The New England Journal of Medicine, vol. 353, no. 16, pp. 1659-1672, 2005.

[22] E. H. Romond, E. A. Perez, J. Bryant et al., "Trastuzumab plus adjuvant chemotherapy for operable HER2-positive breast cancer," The New England Journal of Medicine, vol. 353, no. 16, pp. 1673-1684, 2005.

[23] E. A. Perez, E. H. Romond, V. J. Suman et al., "Four-year follow-up of trastuzumab plus adjuvant chemotherapy for operable human epidermal growth factor receptor 2-positive breast cancer: joint analysis of data from NCCTG N9831 and NSABP B-31," Journal of Clinical Oncology, vol. 29, no. 25, pp. 3366-3373, 2011.

[24] D. Slamon, W. Eiermann, N. Robert et al., "Adjuvant trastuzumab in HER2-positive breast cancer," The New England Journal of Medicine, vol. 365, no. 14, pp. 1273-1283, 2011.

[25] L. Gianni, U. Dafni, R. D. Gelber et al., "Treatment with trastuzumab for 1 year after adjuvant chemotherapy in patients with HER2-positive early breast cancer: a 4-year follow-up of a randomised controlled trial," The Lancet Oncology, vol. 12, no. 3, pp. 236-244, 2011.

[26] H. Joensuu, P. Bono, V. Kataja et al., "Fluorouracil, epirubicin, and cyclophosphamide with either docetaxel or vinorelbine, with or without trastuzumab, as adjuvant treatments of breast cancer: final results of the FinHer trial," Journal of Clinical Oncology, vol. 27, no. 34, pp. 5685-5692, 2009.

[27] H. Joensuu, P. L. Kellokumpu-Lehtinen, P. Bono et al., "Adjuvant docetaxel or vinorelbine with or without trastuzumab for breast cancer," The New England Journal of Medicine, vol. 354, no. 8, pp. 809-820, 2006.

[28] D. J. Slamon, B. Leyland-Jones, S. Shak et al., "Use of chemotherapy plus a monoclonal antibody against her2 for metastatic breast cancer that overexpresses HER2," The New England Journal of Medicine, vol. 344, no. 11, pp. 783-792, 2001.

[29] M. Pegram, S. Hsu, G. Lewis et al., "Inhibitory effects of combinations of HER-2/neu antibody and chemotherapeutic agents used for treatment of human breast cancers," Oncogene, vol. 18 , no. 13, pp. 2241-2251, 1999.

[30] M. Untch, M. Muscholl, S. Tjulandin et al., "First-line trastuzumab plus epirubicin and cyclophosphamide therapy in patients with human epidermal growth factor receptor 2positive metastatic breast cancer: cardiac safety and efficacy data from the herceptin, cyclophosphamide, and epirubicin (HERCULES) trial," Journal of Clinical Oncology, vol. 28, no. 9, pp. 1473-1480, 2010.

[31] M. Marty, F. Cognetti, D. Maraninchi et al., "Randomized phase II trial of the efficacy and safety of trastuzumab combined with docetaxel in patients with human epidermal growth factor receptor 2-positive metastatic breast cancer administered as first-line treatment: the M77001 study group," Journal of Clinical Oncology, vol. 23, no. 19, pp. 4265-4274, 2005.
[32] P. Hamberg, M. M. E. M. Bos, H. J. J. Braun et al., "Randomized phase II study comparing efficacy and safety of combination-therapy trastuzumab and docetaxel vs. sequential therapy of trastuzumab followed by docetaxel alone at progression as first-line chemotherapy in patients with HER2 ${ }^{+}$ metastatic breast cancer: hertax trial," Clinical Breast Cancer, vol. 11, no. 2, pp. 103-113, 2011.

[33] M. Andersson, E. Lidbrink, K. Bjerre et al., "Phase III randomized study comparing docetaxel plus trastuzumab with vinorelbine plus trastuzumab as first-line therapy of metastatic or locally advanced human epidermal growth factor receptor 2-positive breast cancer: the HERNATA study," Journal of Clinical Oncology, vol. 29, no. 3, pp. 264-271, 2011.

[34] V. Valero, J. Forbes, M. D. Pegram et al., "Multicenter phase III randomized trial comparing docetaxel and trastuzumab with docetaxel, carboplatin, and trastuzumab as first-line chemotherapy for patients with HER2-geneamplified metastatic breast cancer (BCIRG 007 Study): two highly active therapeutic regimens," Journal of Clinical Oncology, vol. 29, no. 2, pp. 149-156, 2011.

[35] C. L. Vogel, M. A. Cobleigh, D. Tripathy et al., "Efficacy and safety of trastuzumab as a single agent in first-line treatment of HER2-overexpressing metastatic breast cancer," Journal of Clinical Oncology, vol. 20, no. 3, pp. 719-726, 2002.

[36] B. Kaufman, J. R. Mackey, M. R. Clemens et al., "Trastuzumab plus anastrozole versus anastrozole alone for the treatment of postmenopausal women with human epidermal growth factor receptor 2-positive, hormone receptor-positive metastatic breast cancer: results from the randomized phase III TAnDEM study," Journal of Clinical Oncology, vol. 27, no. 33, pp. 55295537, 2009.

[37] G. Lazaridis, G. Pentheroudakis, and N. Pavlidis, "Integrating trastuzumab in the neoadjuvant treatment of primary breast cancer: accumulating evidence of efficacy, synergy and safety," Critical Reviews in Oncology/Hematology, vol. 66, no. 1, pp. 31-41, 2008.

[38] M. Untch, M. Rezai, S. Loibl et al., "Neoadjuvant treatment with trastuzumab in HER2-positive breast cancer: results from the GeparQuattro study," Journal of Clinical Oncology, vol. 28, no. 12, pp. 2024-2031, 2010.

[39] A. U. Buzdar, N. K. Ibrahim, D. Francis et al., "Significantly higher pathologic complete remission rate after neoadjuvant therapy with trastuzumab, paclitaxel, and epirubicin chemotherapy: results of a randomized trial in human epidermal growth factor receptor 2-positive operable breast cancer," Journal of Clinical Oncology, vol. 23, no. 16, pp. 3676-3685, 2005.

[40] S. A. Limentani, A. M. Brufsky, J. K. Erban, M. Jahanzeb, and D. Lewis, "Phase II study of neoadjuvant docetaxel, vinorelbine, and trastuzumab followed by surgery and adjuvant doxorubicin plus cyclophosphamide in women with human epidermal growth factor receptor 2-overexpressing locally advanced breast cancer," Journal of Clinical Oncology, vol. 25, no. 10, pp. 1232-1238, 2007.

[41] J. Y. Pierga, S. Delaloge, M. Espié et al., "A multicenter randomized phase II study of sequential epirubicin/ cyclophosphamide followed by docetaxel with or without celecoxib or trastuzumab according to HER2 status, as primary chemotherapy for localized invasive breast cancer patients," Breast Cancer Research and Treatment, vol. 122, no. 2, pp. 429437, 2010.

[42] L. Gianni, W. Eiermann, V. Semiglazov et al., "Neoadjuvant chemotherapy with trastuzumab followed by adjuvant trastuzumab versus neoadjuvant chemotherapy alone, in 
patients with HER2-positive locally advanced breast cancer (the NOAH trial): a randomised controlled superiority trial with a parallel HER2-negative cohort," The Lancet, vol. 375, no. 9712, pp. 377-384, 2010.

[43] M. S. Ewer, M. K. Ali, and B. Mackay, "A comparison of cardiac biopsy grades and ejection fraction estimations in patients receiving adriamycin," Journal of Clinical Oncology, vol. 2, no. 2, pp. 112-117, 1984.

[44] M. S. Ewer and S. M. Lippman, "Type II chemotherapy-related cardiac dysfunction: time to recognize a new entity," Journal of Clinical Oncology, vol. 23, no. 13, pp. 2900-2902, 2005.

[45] P. K. Singal, N. Iliskovic, T. Li, and D. Kumar, "Adriamycin cardiomyopathy: pathophysiology and prevention," The FASEB Journal, vol. 11, no. 12, pp. 931-936, 1997.

[46] M. K. Ali, M. S. Ewer, H. R. Gibbs, J. Swafford, and K. L. Graff, "Late doxorubicin-associated cardiotoxicity in children: the possible role of intercurrent viral infection," Cancer, vol. 74, no. 1, pp. 182-188, 1994.

[47] E. T. Yeh, A. T. Tong, D. J. Lenihan et al., "Cardiovascular complications of cancer therapy: diagnosis, pathogenesis, and management," Circulation, vol. 109, no. 25, pp. 3122-3131, 2004.

[48] M. S. Ewer, M. T. Vooletich, J. B. Durand et al., "Reversibility of trastuzumab-related cardiotoxicity: new insights based on clinical course and response to medical treatment," Journal of Clinical Oncology, vol. 23, no. 31, pp. 7820-7826, 2005.

[49] T. M. Suter, N. Cook-Bruns, and C. Barton, "Cardiotoxicity associated with trastuzumab (Herceptin) therapy in the treatment of metastatic breast cancer," Breast, vol. 13, no. 3, pp. 173-183, 2004.

[50] S. A. Crone, Y. Y. Zhao, L. Fan et al., "ErbB2 is essential in the prevention of dilated cardiomyopathy," Nature Medicine, vol. 8, no. 5, pp. 459-465, 2002.

[51] Z. Jiang and M. Zhou, "Neuregulin signaling and heart failure," Current Heart Failure Reports, vol. 7, no. 1, pp. 42-47, 2010.

[52] K. R. Chien, "Stress pathways and heart failure," Cell, vol. 98, no. 5, pp. 555-558, 1999.

[53] L. I. Gordon, M. A. Burke, A. T. K. Singh et al., "Blockade of the erbB2 receptor induces cardiomyocyte death through mitochondrial and reactive oxygen species-dependent pathways," The Journal of Biological Chemistry, vol. 284, no. 4, pp. 2080-2087, 2009.

[54] D. B. Sawyer, C. Zuppinger, T. A. Miller, H. M. Eppenberger, and T. M. Suter, "Modulation of anthracyclineinduced myofibrillar disarray in rat ventricular myocytes by neuregulin- $1 \beta$ and anti-erbB2: potential mechanism for trastuzumab-induced cardiotoxicity," Circulation, vol. 105, no. 13, pp. 1551-1554, 2002.

[55] F. Timolati, D. Ott, L. Pentassuglia et al., "Neuregulin-1 beta attenuates doxorubicin-induced alterations of excitationcontraction coupling and reduces oxidative stress in adult rat cardiomyocytes," Journal of Molecular and Cellular Cardiology, vol. 41, no. 5, pp. 845-854, 2006.

[56] Y. Y. Zhao, D. R. Sawyer, R. R. Baliga et al., "Neuregulins promote survival and growth of cardiac myocytes: persistence of ErbB2 and ErbB4 expression in neonatal and adult ventricular myocytes," The Journal of Biological Chemistry, vol. 273, no. 17, pp. 10261-10269, 1998.

[57] M. Zeglinski, A. Ludke, D. S. Jassal, and P. K. Singal, "Trastuzumab-induced cardiac dysfunction: a 'dual-hit," Experimental and Clinical Cardiology, vol. 16, no. 3, pp. 70-74, 2011.
[58] D. Cardinale, A. Colombo, M. T. Sandri et al., "Prevention of high-dose chemotherapy-induced cardiotoxicity in highrisk patients by angiotensin-converting enzyme inhibition," Circulation, vol. 114, no. 23, pp. 2474-2481, 2006.

[59] H. Nakagami, M. Takemoto, and J. K. Liao, "NADPH oxidasederived superoxide anion mediates angiotensin II-induced cardiac hypertrophy," Journal of Molecular and Cellular Cardiology, vol. 35, no. 7, pp. 851-859, 2003.

[60] L. P. Grazette, W. Boecker, T. Matsui et al., "Inhibition of ErbB2 causes mitochondrial dysfunction in cardiomyocytes: implications for herceptin-induced cardiomyopathy," Journal of the American College of Cardiology, vol. 44, no. 11, pp. 22312238, 2004.

[61] J. N. Cohn, R. Ferrari, and N. Sharpe, "Cardiac remodelingconcepts and clinical implications: a consensus paper from an International Forum on Cardiac Remodeling," Journal of the American College of Cardiology, vol. 35, no. 3, pp. 569-582, 2000.

[62] L. Pentassuglia, M. Graf, H. Lane et al., "Inhibition of ErbB2 by receptor tyrosine kinase inhibitors causes myofibrillar structural damage without cell death in adult rat cardiomyocytes," Experimental Cell Research, vol. 315, no. 7, pp. 1302-1312, 2009.

[63] M. Procter, T. M. Suter, E. De Azambuja et al., "Longerterm assessment of trastuzumab-related cardiac adverse events in the Herceptin Adjuvant (HERA) trial," Journal of Clinical Oncology, vol. 28, no. 21, pp. 3422-3428, 2010.

[64] M. S. Ewer and J. A. O'Shaughnessy, "Cardiac toxicity of trastuzumab-related regimens in HER2-overexpressing breast cancer," Clinical Breast Cancer, vol. 7, no. 8, pp. 600-607, 2007.

[65] N. Robert, B. Leyland-Jones, L. Asmar et al., "Randomized phase III study of trastuzumab, paclitaxel, and carboplatin compared with trastuzumab and paclitaxel in women with HER-2-overexpressing metastatic breast cancer," Journal of Clinical Oncology, vol. 24, no. 18, pp. 2786-2792, 2006.

[66] D. Cardinale, A. Colombo, R. Torrisi et al., "Trastuzumabinduced cardiotoxicity: clinical and prognostic implications of troponin I evaluation," Journal of Clinical Oncology, vol. 28, no. 25, pp. 3910-3916, 2010.

[67] D. Cardinale, M. T. Sandri, A. Martinoni et al., "Left ventricular dysfunction predicted by early troponin I release after high-dose chemotherapy," Journal of the American College of Cardiology, vol. 36, no. 2, pp. 517-522, 2000.

[68] D. L. Mann and M. R. Bristow, "Mechanisms and models in heart failure: the biomechanical model and beyond," Circulation, vol. 111, no. 21, pp. 2837-2849, 2005.

[69] H. L. McArthur and S. Chia, "Cardiotoxicity of trastuzumab in clinical practice," The New England Journal of Medicine, vol. 357, no. 1, pp. 94-95, 2007.

[70] M. L. Telli, S. A. Hunt, R. W. Carlson, and A. E. Guardino, "Trastuzumab-related cardiotoxicity: calling into question the concept of reversibility," Journal of Clinical Oncology, vol. 25, no. 23, pp. 3525-3533, 2007.

[71] N. Kalay, E. Basar, I. Ozdogru et al., "Protective effects of carvedilol against anthracycline-induced cardiomyopathy," Journal of the American College of Cardiology, vol. 48, no. 11, pp. 2258-2262, 2006.

[72] E. Pituskin, M. Haykowsky, J. R. Mackey et al., "Rationale and design of the Multidisciplinary Approach to Novel Therapies in Cardiology Oncology Research Trial (MANTICORE 101Breast): a randomized, placebo-controlled trial to determine if conventional heart failure pharmacotherapy can prevent trastuzumab-mediated left ventricular remodeling among 
women with $\mathrm{HER}^{+}$early breast cancer using cardiac MRI," BMC Cancer, vol. 11, article 318, 2011.

[73] M. Scaltriti, F. Rojo, A. Ocaña et al., "Expression of p95HER2, a truncated form of the HER2 receptor, and response to Anti-HER2 therapies in breast cancer," Journal of the National Cancer Institute, vol. 99, no. 8, pp. 628-638, 2007.

[74] W. Xia, L. H. Liu, P. Ho, and N. L. Spector, "Truncated ErbB2 receptor (p95 ErbB2 $)$ is regulated by heregulin through heterodimer formation with ErbB3 yet remains sensitive to the dual EGFR/ErbB2 kinase inhibitor GW572016," Oncogene, vol. 23, no. 3, pp. 646-653, 2004.

[75] M. W. Karaman, S. Herrgard, D. K. Treiber et al., "A quantitative analysis of kinase inhibitor selectivity," Nature Biotechnology, vol. 26, no. 1, pp. 127-132, 2008.

[76] D. W. Rusnak, K. Lackey, K. Affleck et al., "The effects of the novel, reversible epidermal growth factor receptor/ErbB-2 tyrosine kinase inhibitor, GW2016, on the growth of human normal and tumor-derived cell lines in vitro and in vivo," Molecular Cancer Therapeutics, vol. 1, no. 2, pp. 85-94, 2001.

[77] G. E. Konecny, M. D. Pegram, N. Venkatesan et al., "Activity of the dual kinase inhibitor lapatinib (GW572016) against HER2-overexpressing and trastuzumab-treated breast cancer cells," Cancer Research, vol. 66, no. 3, pp. 1630-1639, 2006.

[78] D. Cameron, M. Casey, M. Press et al., "A phase III randomized comparison of lapatinib plus capecitabine versus capecitabine alone in women with advanced breast cancer that has progressed on trastuzumab: updated efficacy and biomarker analyses," Breast Cancer Research and Treatment, vol. 112, no. 3, pp. 533-543, 2008.

[79] K. L. Blackwell, H. J. Burstein, A. M. Storniolo et al., "Randomized study of lapatinib alone or in combination with trastuzumab in women with ErbB2-positive, trastuzumabrefractory metastatic breast cancer," Journal of Clinical Oncology, vol. 28, no. 7, pp. 1124-1130, 2010.

[80] S. Johnston, J. Pippen, X. Pivot et al., "Lapatinib combined with letrozole versus letrozole and placebo as first-line therapy for postmenopausal hormone receptor-positive metastatic breast cancer," Journal of Clinical Oncology, vol. 27, no. 33, pp. 5538-5546, 2009.

[81] D. B. Agus, R. W. Akita, W. D. Fox et al., "Targeting ligandactivated ErbB2 signaling inhibits breast and prostate tumor growth," Cancer Cell, vol. 2, no. 2, pp. 127-137, 2002.

[82] J. Baselga and S. M. Swain, "CLEOPATRA: a phase III evaluation of pertuzumab and trastuzumab for HER2-positive metastatic breast cancer," Clinical Breast Cancer, vol. 10, no. 6, pp. 489-491, 2010.

[83] J. Baselga, J. Cortés, S.-B. Kim et al., "Pertuzumab plus trastuzumab plus docetaxel for metastatic breast cancer," The New England Journal of Medicine, vol. 366, no. 2, pp. 109-119, 2012. 


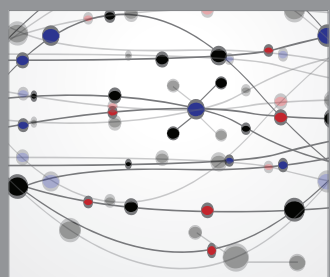

The Scientific World Journal
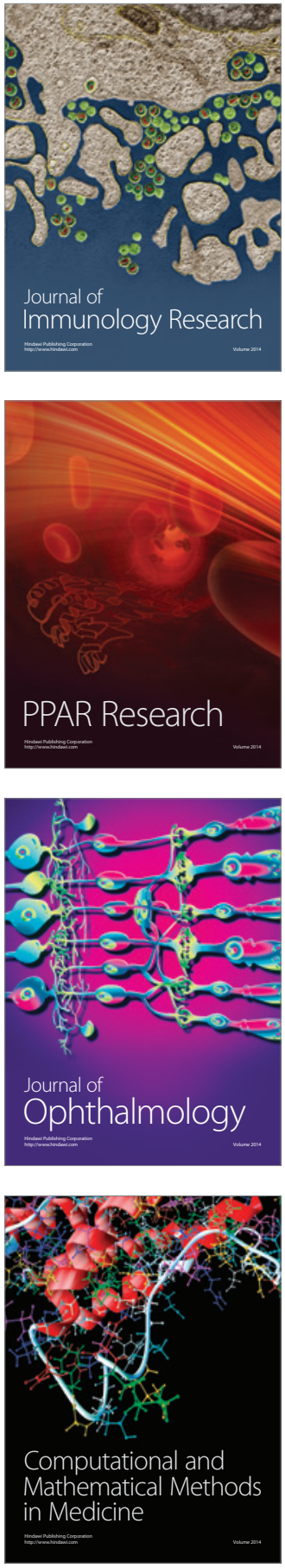

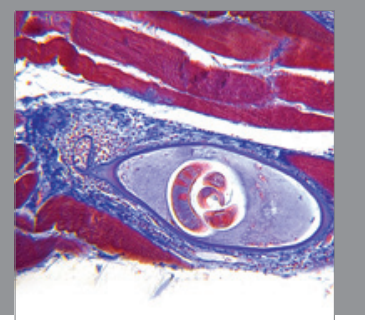

Gastroenterology

Research and Practice
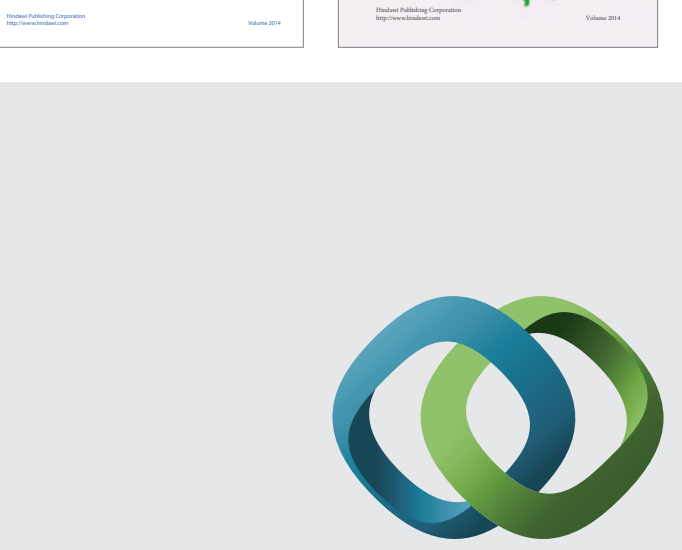

\section{Hindawi}

Submit your manuscripts at

http://www.hindawi.com
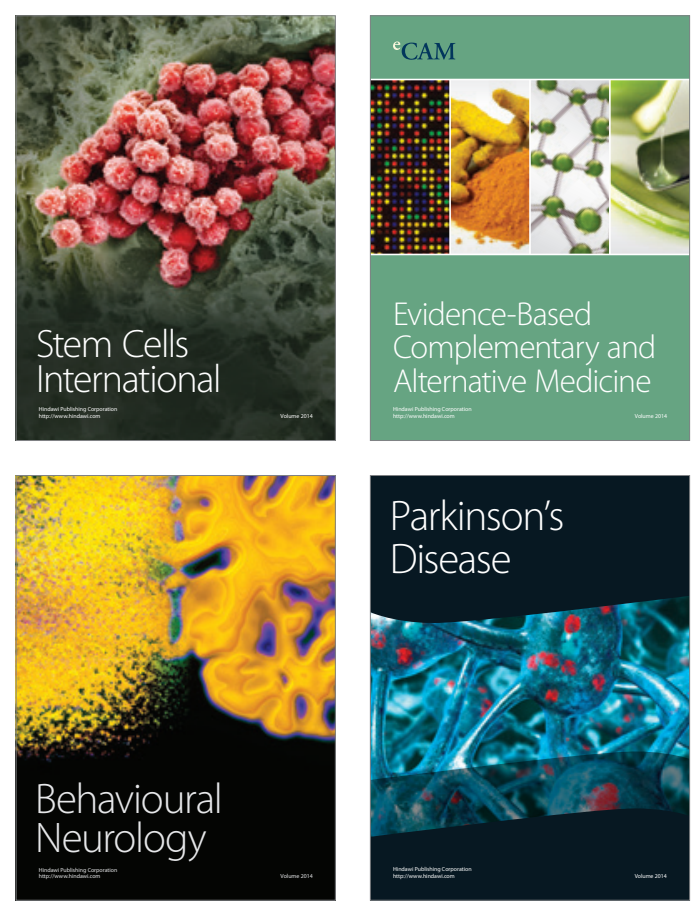

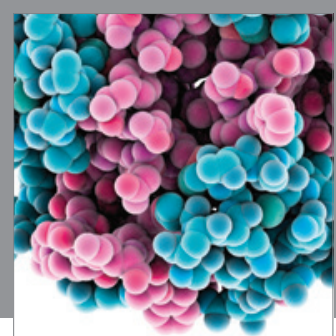

Journal of
Diabetes Research

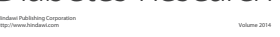

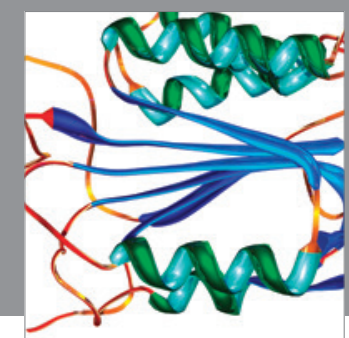

Disease Markers
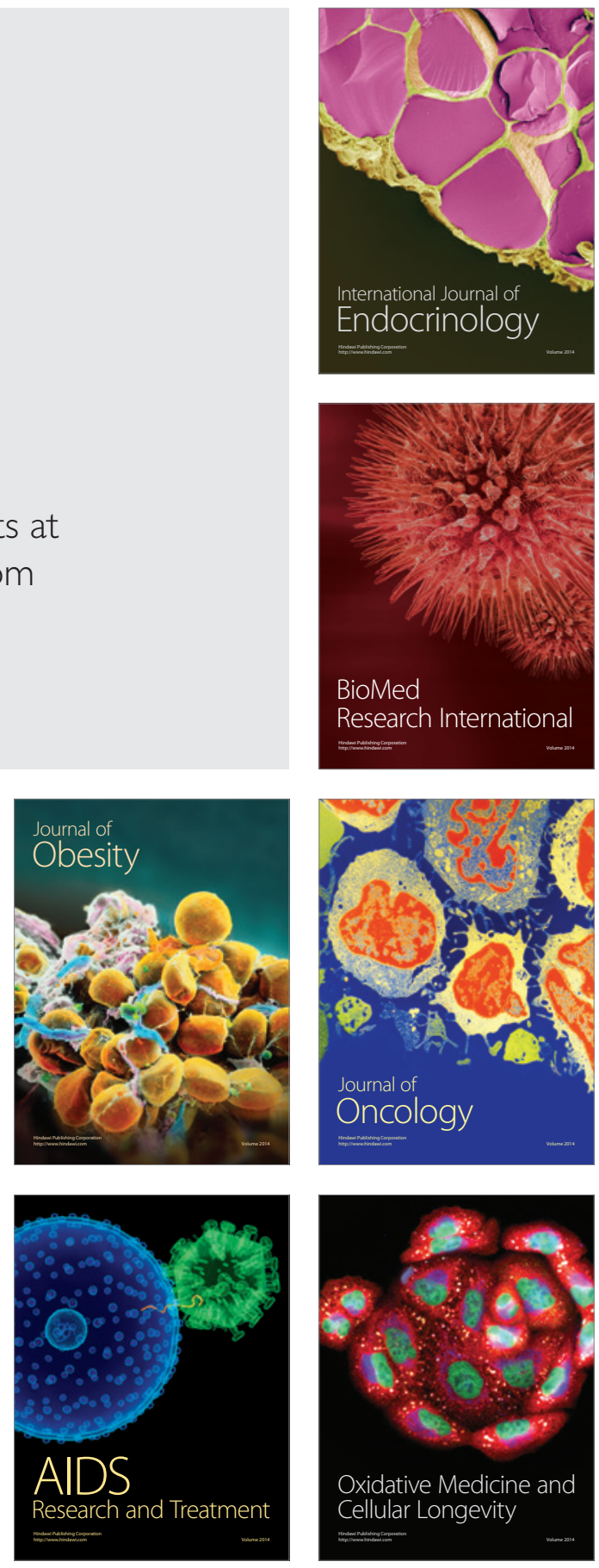